\title{
Potential Biomarker for Fatty Liver Hemorrhagic Syndrome in Laying Hens
}

\author{
Lihui Zhu ${ }^{1,2^{*}}$, Rongrong Liao ${ }^{1}$, Changfeng Xiao ${ }^{1}$, Gensheng $\mathrm{Zhu}^{2,3}$, Ning $\mathrm{Wu}^{3}$, Yinyin $\mathrm{Tu}^{2}$, and Changsuo Yang ${ }^{1,2 * *}$ \\ ${ }^{1}$ Institute of Animal Husbandry and Veterinary Science, Shanghai Academy of Agricultural Sciences, Shanghai, 201106, China \\ ${ }^{2}$ National Poultry Research Center for Engineering and Technology, Shanghai, 201106, China \\ ${ }^{3}$ Shanghai Haifeng Dafeng Poultry CO. LTD., Bright Food (Group) CO, LTD., Shanghai, 200085, China \\ "Corresponding author's Email: zhulihui@saas.sh.cn; ORCID: 0000-0002-2931-6207 \\ *** Corresponding author's Email: yangchangsuo@189.cn; ORCID: 0000-0002-6285-3020
}

Received: 09 Oct. 2020

Accepted: 20 Dec. 2020

\begin{abstract}
Fatty liver hemorrhagic syndrome is more common in laying hens with excess body weight $(\mathrm{BW})$ and in the middle and late phase of egg production. However, no specific biomarkers in chickens can be used to diagnose liver steatosis or liver injury. The present study aimed to assess whether BW can be used to predict fatty liver in aged laying hens. This study also searched for potential plasma FLHS biomarkers. For these purposes, correlation among BW, relative weight of liver and abdominal fat, and plasma markers were analyzed in Hy-line brown laying hens. Furthermore, plasma levels of potential biomarkers were analyzed during the formation of fatty liver. Concentrations of triglycerides and total cholesterol were positively associated with BW in aged laying hens, while liver fat deposition was similar among chickens with different $\mathrm{BW}$, indicating that $\mathrm{BW}$ cannot be used as the only criterion to discriminate aged laying hens with liver steatosis. A trend of increasing triglyceride, total cholesterol, fatty acidbinding protein 4 (FABP4), and lipoprotein lipase levels was found as age increased, and they were positively associated with BW indicating that they might be risk markers for FLHS in laying hens. The findings indicated that the plasma level of FABP4 was positively associated with the severity of fatty liver in aged laying hens. All the above results suggested that FABP4 might be a potential diagnostic indicator for FLHS.
\end{abstract}

Key words: Biomarker, Egg production, Fatty liver, Laying hens, Poultry

\section{INTRODUCTION}

Similar to nonalcoholic fatty liver disease (NAFLD), fatty liver hemorrhagic syndrome (FLHS) is related to liver degeneration resulted from excessive fat deposition in the liver causing liver rupture, hemorrhage, and sudden death of chickens (Wolford and Polin, 1974; Whitehead 1979). Excessive fat deposition is also observed in the abdominal cavity. This disease mainly occurs in commercial caged laying hens leading to reduced egg production and death, and accordingly significant economic losses (Julian, 2005). Nowadays, FLHS is the most common noninfectious cause of high mortality in laying hens (Trott et al., 2014). It is currently believed that nutrition, genetics, and environmental toxins are related to FLHS outbreaks in laying hen farms, and the low-protein and high-energy diet is the main reason for the occurrence of FLHS (Diaz et al., 1999; Choi et al., 2012; Rozenboim et al., 2016). There are no obvious symptoms in the early stage of the disease, and the diagnosis can only be confirmed by dissecting the chickens with sudden onset. Therefore, screening of specific biomarkers for liver steatosis or liver damage would help improve the quality and efficiency of large-scale farmed laying hens.

Conventionally, FLHS is more prevalent in birds with excess body weight (BW) and in the middle and late phases of egg production. However, the idea of whether BW can be used as a marker for FLHS has remained to be verified. Additionally, in birds, the liver is the major organ of lipid metabolism, and steady changes in liver lipid metabolism are the basis of various forms of fatty liver disease. Excess fatty acid supply or inhibited oxidation in the liver may result in enhanced synthesis of triglycerides (TG) and disordered very low-density lipoprotein (VLDL) synthesis and secretion, which are observed in laying hens with FLHS (Dong and Tong, 2019; Gao et al., 2019). Circulating alanine aminotransferase (ALT) and aspartate aminotransferase (AST) are used as liver injury indicators and have been found to be increased in laying hens with 
FLHS (Hamid et al., 2019). Furthermore, many proteins in the blood, including those related to lipid metabolism, are synthesized in the liver, and the abundance and structure of these proteins also change in the development of liver disease, making them potential biomarkers for liver diseases including NAFLD (Kim et al., 2011; VilarGomez and Chalasani, 2018). However, the clinical utility of these biomarkers has not been validated in chicken cohorts. The objectives of this study were, first, to assess whether BW can be used as an indicator for predicting fatty liver disease, and second, to screen potential plasma FLHS biomarkers in chickens.

\section{MATERIALS AND METHODS}

\section{Ethical approval}

All animal protocols used in this study were approved by the Ethics and Animal Welfare Committee of Shanghai Academy of Agricultural Sciences, China, and performed according to the guidelines established by 'the instructive notions with respect to caring for laboratory animals' issued by the Ministry of Science and Technology of the People's Republic of China (No. [2006] 398).

\section{Animals and study design}

A total number of 102,000 commercial Hy-line brown laying hens (20 to 65 weeks old) were raised in eight-high modular manure belt cages (Chore-Time Equipment Inc., Milford, IN, USA), with nine chickens per cage (dimensions of $60 \times 65 \times 45.5 \mathrm{~cm}$ ) under standard commercial conditions. A standard commercial corn-soy diet (Shanghai Haifeng Dafeng Poultry Co. Ltd. Dafeng, Jiangsu, China) containing $16.00 \%$ crude protein, $0.73 \%$ lysine, $0.33 \%$ methionine, $4.1 \%$ calcium, $0.36 \%$ phosphorus, $0.17 \%$ salt, 19.3 ppm copper, $66 \mathrm{ppm}$ iron, 80 ppm manganese, $0.3 \mathrm{ppm}$ selenium, $2.2 \mathrm{ppm}$ iodine, 80 $\mathrm{ppm}$ zinc, and $2700 \mathrm{kcal} / \mathrm{kg}$ calculated metabolizable energy was supplied for the layers. Feed and water were offered ad libitum throughout the experimental period. Separate groups of chickens were used for each experiment (Experiments 1-3).

\section{Experiment 1}

To verify whether BW can be used as a potential predictor of FLHS in aged laying hens, 60 laying hens aged 52 weeks were randomly divided into 4 groups according to the BW recommended by the Hy-line brown laying hens management guide (http://www.hyline.co.uk/services/management-guides/). These four groups included low-grade weight (LW, 5\% below standard weight), normal weight ( $\mathrm{NW}$, in standard weight range), overweight/obese $(\mathrm{OW} / \mathrm{Ob},<5 \%$ over standard weight), and seriously overweight/obese (SW/Ob, 5-10\% over standard weight). The standard BW of Hy-line brown laying hens at 52 weeks should be 1.89 to $2.01 \mathrm{~kg}$. To determine plasma concentrations of ALT, AST, TG, total cholesterol (TC), VLDL cholesterol (VLDL-C), fatty acidbinding protein 4 (FABP4), and lipoprotein lipase (LPL) blood samples from the experimental layers were obtained through the wing vein and collected in tubes containing EDTA as an anticoagulant. About 4 hours later, all the hens were killed by electric shock, and the abdominal fat and livers were removed and weighed immediately. The collected liver samples were immediately snap-frozen in liquid nitrogen and stored at $-80{ }^{\circ} \mathrm{C}$ for expression analysis of lipid metabolism-related genes. Another portion of the liver was fixed in $4 \%$ paraformaldehyde for histological analysis. The relative weight of abdominal fat and liver was calculated as the weight of abdominal fat or liver in $100 \mathrm{~g}$ body weight $(\mathrm{g} / 100 \mathrm{~g} \mathrm{BW})$. The severity of fatty liver was evaluated by liver color and scored, as modified based on a study conducted by Choi et al. (2012). The fatty liver was scored on a scale from 1 to 4 as follows: FS1, normal liver with dark red; FS2, mild case of FLHS with mild yellow liver and hemorrhages; FS3, moderate case of FLHS with light yellowish red liver and hemorrhages; and FS4, large and massive hemorrhages with putty-colored livers. The standard for the fatty liver score according to Avinash (2007) is indicated in Figure 1.

\section{Experiment 2}

To assess mRNA expression of lipid metabolismrelated genes, a total of 30 laying hens were killed by electric shock at specified ages ( 15 at the age of 27 weeks and 15 at the age of 52 weeks). All livers were harvested and checked for fatty liver by gross examination. The livers with dark red color and no hemorrhages were determined as normal liver and livers with light yellowish red color and hemorrhages were considered as fatty liver. From each group (normal and fatty liver), 4-5 samples were pooled together for mRNA expression analysis of some lipid metabolism-related genes, including apolipoprotein B (APOB), peroxisome proliferatoractivated receptor $\alpha(P P A R \alpha), P P A R \gamma, L P L$, and FABP4.

\section{Experiment 3}

To determine the changes in concentrations of some biochemical indicators, including TG, TC, ALT, AST, FABP4, and LPL, with age, blood samples from 40 laying hens were collected every 5 weeks from age 27 to 52 weeks. At age of 52 weeks, all 40 laying hens were killed by electric shock, and checked for fatty liver as described above. 


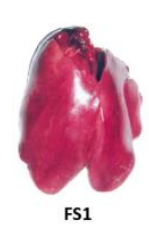

Normal liver Dark red
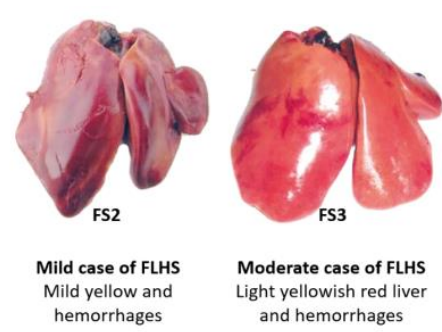
and hemorrhages

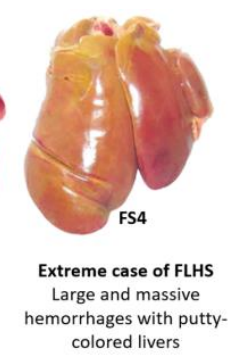

Figure 1. The standard for fatty liver score used for fatty liver examination in experiments 1-3. The color score, from 1 to 4 (from dark red to light yellowish-red), was judged by three investigators.

\section{Histological analysis}

The paraffin-embedded liver was cut into $5-\mu \mathrm{M}$ sections and stained with hematoxylin and eosin. The degree of lipid accumulation was evaluated by the deposition of lipid droplets, which was quantified by ImageJ software (version 1.80, National Institutes of Health, Bethesda, MD, USA). The lipid accumulation was represented by the deposition of lipid droplets, with white pixel areas indicating fat vacuoles being divided by the total area scanned. The ImageJ software was used to quantify the staining of the liver section.

\section{Biochemical analysis}

Plasma samples were prepared by centrifugation (3,000 g for $15 \mathrm{~min}$ ) and stored at $-20{ }^{\circ} \mathrm{C}$ for future analysis. Concentrations of TG, TC, ALT, and AST were measured by colorimetric endpoint assays (A110-1-1, A111-1-1, C009-2-1, and C010-1-1 kits, respectively; Nanjing Jiancheng Bioengineering Institute, Nanjing, China). Total concentrations of plasma FABP4 and LPL were determined with chicken-specific commercial ELISA kits (Shanghai BlueGene Biotech Co. Ltd., China). Results were analyzed using the Thermo Multiskan Sky microplate reader (Thermo Fisher, Waltham, MA, USA). For liver TG and TC analysis, total proteins of liver were measured by bicinchoninic acid assay (kit A045-4-2; Nanjing Jiancheng Bioengineering Institute, Nanjing, China), the contents of liver TG and TC were measured by using kits A110-1-1 and A111-1-1, respectively (Nanjing Jiancheng Bioengineering Institute, Nanjing, China). All assays were performed according to the manufacturer's instruction.

\section{Gene expression analysis}

Chicken liver RNA was extracted using TRIzol Reagent (Invitrogen, Carlsbad, CA, USA) and checked on a Nanotrop Bioanalyzer (Agilent 2100; Palo Alto, CA,
USA) for integrity analysis. The mRNA expression was detected by RT-qPCR in the liver. A PrimeScript RT reagent Kit (TaKaRa Biotechnology Co., Japan) was used to reverse transcribe RNA to cDNA, and qPCR was performed with SYBR Premix Ex Taq (TaKaRa Biotechnology Co., Japan) using an ABI Q5 Real-time PCR instrument (Applied Biosystems, Foster City, USA) at $95{ }^{\circ} \mathrm{C}$ for $30 \mathrm{~s}$, followed by 35 cycles of $95{ }^{\circ} \mathrm{C}$ for $5 \mathrm{~s}$, and $60{ }^{\circ} \mathrm{C}$ for $30 \mathrm{~s}$. For RT-qPCR data analysis, each sample was run in triplicate. The mRNAs were normalized to $G A P D H$ mRNA. The primer sequences are shown in Table 1. Data were analyzed using the $2^{-\Delta \Delta \mathrm{Ct}}$ method (Livak and Schmittgen, 2001).

\section{Statistical analysis}

Statistical analyses were carried out using SPSS version 21 (SPSS Inc., Chicago, IL, USA). Data were analyzed using one-way ANOVA and a post hoc Tukey test. P-value less than 0.05 was considered statistically significant. Spearman's correlation coefficient was used to test the relationship between various variables.

Table 1. Primers used in this study for RT-qPCR

\begin{tabular}{ll}
\hline Gene & Sequence (5'-3') \\
\hline$\beta$-actin-F & AATGGCTCCGGTATGTGCAA \\
$\beta$-actin-R & GGCCCATACCAACCATCACA \\
GAPDH-F & GATGGGTGTCAACCATGAGAAA \\
GAPDH-R & CAATGCCAAAGTTGTCATGGA \\
PPAR $\alpha-F$ & GCTTGTGAAGGTTGTAAGGGTT \\
PPAR $\alpha-R$ & GACATTCCAACTGAAAGGCAC \\
PPAR $\gamma-F$ & GTCCTTCCCGCTGACCAAA \\
PPAR $\gamma-R$ & TCTCCTGCACTGCCTCCACA \\
FABP4-F & GGCCAAGCCTAATTTAACTATC \\
FABP4-R & TCCCATCCACCACTTTTCTC \\
APOB-F & ATACCCTGGGACTCTTGCCT \\
APOB-R & GAGAAGCTTTCAGGCTGGGT \\
LPL-F & ATTGCTGCCTCTTCTCCTTT \\
LPL-R & ATTGGTGACCTGCTTATGCTA \\
\hline GAPDH: glyceraldehyde-3-phosphate dehydrogenase; PPAR $:$ \\
peroxisome proliferator-activated receptor $\alpha$, PPAR $\gamma:$ peroxisome \\
proliferator-activated receptor $\gamma ;$ FABP4: fatty acid-binding protein 4; \\
APOB: apolipoprotein B; LPL: lipoprotein lipase.
\end{tabular}

\section{RESULTS}

\section{Experiment 1}

\section{Liver and abdominal fat weight}

In Experiment 1, different groups were significantly different in terms of BW $(\mathrm{P}<0.05$, Table 2). The amount of abdominal fat sequentially increased in chickens with 
the increase of $\mathrm{BW}$, although there was no significant difference between the normal $\mathrm{BW}$ and $\mathrm{OW} / \mathrm{Ob}$ group $(\mathrm{P}$ $>0.05)$. The relative weight of abdominal fat was similar in both low and normal BW hens, whereas a significant increase of the relative weight of abdominal fat was observed in the overweight chickens (OW/Ob and SW/Ob groups), compared to the low BW group ( $\mathrm{P}<0.05)$. The relative weight of abdominal fat increased in the $\mathrm{SW} / \mathrm{Ob}$ group, compared to the $\mathrm{OW} / \mathrm{Ob}$ group $(\mathrm{P}<0.05)$. There was no significant difference in the relative liver weight among chickens with different BW $(\mathrm{P}>0.05)$. The concentration of TG was significantly higher in the $\mathrm{SW} / \mathrm{Ob}$ hens, compared to the LW and the NW groups, while the obtained values of $\mathrm{LW}, \mathrm{NW}$, and $\mathrm{OW} / \mathrm{Ob}$ hens were similar. The concentrations of TC were significantly lower in the LW laying hens than in the overweight hens $(\mathrm{OW} / \mathrm{Ob}$ or $\mathrm{SW} / \mathrm{Ob}$ ), and the levels of AST were significantly increased in the NW, OW/Ob, and the SW/
Ob groups in comparison to the LW hens. No significant differences in VLDL-C were observed among the four groups.

Correlation of body weight, relative weight of liver and abdominal fat with plasma markers

The abdominal fat pad weight, concentrations of TC and TG were significantly associated with BW $(\mathrm{P}<0.05$, Table 3). The concentrations of TC and TG were also positively correlated with the abdominal fat pad weight. The correlation between plasma TG and BW $(r=0.662)$ was stronger than that between plasma ALT and BW $(r=$ $0.108)$, or plasma TC and BW $(r=0.513)$. Although other biomarkers were associated with each other (TG vs. ALT, $\mathrm{r}=0.224$; TG vs. TC, $\mathrm{r}=0.297$; VLDL-C vs. abdominal fad pad weight, $r=0.169$ ), their correlations were insignificant. There was no significant correlation between liver weight and other biomarkers.

Table 2. Chicken body weights, relative weights of liver and abdominal fat, and plasma markers in the various body weight groups (Experiment 1)

\begin{tabular}{|c|c|c|c|c|}
\hline Parameters & $\mathbf{L W}(\mathbf{n}=\mathbf{1 5})$ & NW (n=15) & OW/Ob $(n=15)$ & $\mathrm{SW} / \mathrm{Ob}(\mathrm{n}=15)$ \\
\hline BW (g) & $1665.71 \pm 40.03^{\mathrm{a}}$ & $1956.70 \pm 19.68^{b}$ & $2084.43 \pm 14.17^{\mathrm{c}}$ & $2291.59 \pm 40.95^{\mathrm{d}}$ \\
\hline \multicolumn{5}{|c|}{ Relative weight, g/100 g BW } \\
\hline Abdominal fat & $2.49 \pm 0.21^{\mathrm{a}}$ & $3.05 \pm 0.27^{\mathrm{ab}}$ & $3.99 \pm 0.38^{\mathrm{b}}$ & $4.94 \pm 0.37^{\mathrm{c}}$ \\
\hline Liver & $1.96 \pm 0.07$ & $2.11 \pm 0.08$ & $1.98 \pm 0.11$ & $2.16 \pm 0.17$ \\
\hline \multicolumn{5}{|l|}{ Plasma markers } \\
\hline ALT(U/L) & $17.03 \pm 2.68$ & $11.36 \pm 1.43$ & $21.48 \pm 6.63$ & $25.41 \pm 5.01$ \\
\hline AST(U/L) & $26.97 \pm 1.33^{\mathrm{a}}$ & $36.14 \pm 1.94^{\mathrm{b}}$ & $33.95 \pm 2.70^{\mathrm{b}}$ & $35.57 \pm 1.88^{\mathrm{b}}$ \\
\hline $\mathrm{TC}(\mathrm{mmol} / \mathrm{L})$ & $2.13 \pm 0.26^{\mathrm{a}}$ & $3.01 \pm 0.19^{\mathrm{ab}}$ & $4.13 \pm 0.50^{\mathrm{b}}$ & $3.57 \pm 0.27^{\mathrm{b}}$ \\
\hline VLDL-C (mmol/L) & $2.25 \pm 0.21$ & $3.64 \pm 0.50$ & $2.95 \pm 0.34$ & $3.34 \pm 0.76$ \\
\hline $\mathrm{TG}(\mathrm{mmol} / \mathrm{L})$ & $13.45 \pm 1.10^{\mathrm{a}}$ & $16.58 \pm 1.20^{\mathrm{a}}$ & $23.17 \pm 2.09^{\mathrm{ab}}$ & $30.21 \pm 3.09^{\mathrm{b}}$ \\
\hline
\end{tabular}

Table 3. Spearman correlation coefficients among the chicken body weights, relative weights of liver and abdominal fat, and serum markers (Experiment 1).

\begin{tabular}{|c|c|c|c|c|c|c|c|}
\hline \multirow[b]{2}{*}{ Variables } & \multirow[b]{2}{*}{ TG } & \multirow[b]{2}{*}{ TC } & \multirow[b]{2}{*}{ VLDL-C } & \multirow[b]{2}{*}{ AST } & \multirow[b]{2}{*}{ ALT } & \multicolumn{2}{|c|}{ Relative weight, g/100 g BW } \\
\hline & & & & & & $\begin{array}{c}\text { Abdominal fat } \\
\text { weight }\end{array}$ & Liver weight \\
\hline BW & $0.662^{* *}$ & $0.513^{* *}$ & 0.235 & 0.248 & 0.108 & $0.658^{* *}$ & 0.085 \\
\hline TG & & 0.297 & 0.097 & 0.152 & 0.224 & $0.318^{*}$ & 0.057 \\
\hline $\mathrm{TC}$ & & & -0.024 & 0.299 & 0.018 & $0.349^{* *}$ & 0.075 \\
\hline VLDL-C & & & & 0.142 & -0.093 & 0.169 & -0.107 \\
\hline AST & & & & & -0.257 & 0.257 & -0.003 \\
\hline ALT & & & & & & -0.033 & 0.057 \\
\hline $\begin{array}{l}\text { Relative abdominal fat weight, } \\
\mathrm{g} / 100 \mathrm{~g} \mathrm{BW}\end{array}$ & & & & & & & -0.187 \\
\hline
\end{tabular}

BW: Body weight, TG: Triglyceride, TC: Total cholesterol, VLDL-C: Very low-density lipoprotein cholesterol, AST: Aspartate aminotransferase, ALT: Alanine aminotransferase. ${ }^{*} \mathrm{P}<0.05,{ }^{*} \mathrm{P}<0.01$. 


\section{Hepatic lipid accumulation}

Pathological changes in the liver in all four groups at age 52 weeks are presented in Figure 2. The livers of overweight hens (OW/Ob and $\mathrm{SW} / \mathrm{Ob}$ groups) were fragile and yellow, and some hemorrhagic spots appeared due to the high lipid accumulation when compared to layers with low BW (Figure 2a, b). The fatty liver score increased with BW. There was a significant increase in fatty liver score in the overweight groups (OW/Ob and SW/Ob groups), compared to the LW group $(\mathrm{P}<0.05)$, while the fatty liver score in overweight hens $(\mathrm{OW} / \mathrm{Ob}$ and $\mathrm{SW} / \mathrm{Ob}$ groups) showed no significant difference between the two groups $(\mathrm{P}>0.05$, Figure 2c). However, the liver turned light yellow in the $\mathrm{OW} / \mathrm{Ob}$ and $\mathrm{SW} / \mathrm{Ob}$ groups indicating an increased fatty liver score, compared to the low and normal BW groups. To evaluate the consistency between liver lipid deposition and BW, the vacuolar areas of liver sections were quantified using image $\mathrm{J}$ software. The vacuolar area in the livers from overweight chickens (OW/Ob and $\mathrm{SW} / \mathrm{Ob}$ groups) was significantly larger, compared to the low and normal BW groups $(\mathrm{P}<0.05)$. No significant difference was observed between the two overweight groups (Figure 2d, $\mathrm{P}>0.05$ ). Concentrations of liver TG and TC were not significantly different in the chicken livers from the four groups $(\mathrm{P}>0.05$, Figure $2 \mathrm{e}$ ).

\section{Expression of lipid metabolism-related genes}

The RT-qPCR analysis of lipid metabolism-related genes confirmed the changes in expression among the chickens with different levels of BW (Figure 3a). However, no regular expression of these genes was found among the layers with different BW values. As BW increased, $A P O B$ expression gradually decreased, and the mRNA expression of $A P O B$ decreased in the $\mathrm{SW} / \mathrm{Ob}$ group compared to the LW group $(\mathrm{P}<0.05)$, while it was not significantly different among the NW, OW/Ob, and $\mathrm{SW} / \mathrm{Ob}$ groups $(\mathrm{P}>0.05)$. Expression of PPAR $\alpha$ was higher in the NW group, compared to the $\mathrm{LW}, \mathrm{OW} / \mathrm{Ob}$, and SW/Ob groups $(\mathrm{P}<0.05)$, however, no significant differences were observed among the three groups $(\mathrm{P}>$ 0.05). The mRNA expression of FABP4 increased significantly in the NW, OW/Ob, and SW/Ob groups $(\mathrm{P}<$ 0.05), compared to the LW group, with the highest expression in the NW group. The expression of PPAR $\gamma$ was highest in the SW/Ob group, whereas there was no significant difference among the other three groups ( $\mathrm{P}>$ 0.05). There was no significant difference in $L P L$ mRNA expression among the four groups $(\mathrm{P}>0.05)$ although it tended to increase with BW.

\section{Plasma concentrations of FABP4 and LPL}

Plasma concentrations of FABP4 and LPL in laying hens with different BW were also measured (Figure $3 b$ ). The concentration of FABP4 significantly increased as BW increased at age 52 weeks although no significant changes in LPL levels were observed among the four groups with different BW values. Plasma concentration of FABP4, measured by ELISA, was significantly upregulated and positively correlated with the fatty liver score (Figure 5c), indicating the diagnostic potential of plasma concentrations of FABP4. No significant correlation was found between plasma LPL and fatty liver score (data not shown).

\section{Experiment 2}

Increase of plasma FABP4 and LPL levels in chickens with fatty liver

To screen potential biomarkers in this chicken model of FLHS, five known lipid metabolism-related genes, including $A P O B, P P A R \alpha, P P A R \gamma, L P L$, and FABP4 were selected for analyzing mRNA expression in normal and fatty livers. All samples from the laying hens of 52 weeks old were fatty liver. In laying hens of 27 weeks old, 12 out of 15 liver samples were normal; 3 liver with mild yellow color and hemorrhages were discarded from mRNA analysis. The results indicated that mRNA expression of $F A B P 4$ and $L P L$ significantly increased $(\mathrm{P}<0.05)$, while the expression of $A P O B, P P A R \alpha$, and PPAR $\gamma$ decreased (P $<0.05$ ) in fatty livers, compared to normal livers (Figure $3 c)$.

\section{Experiment 3}

Evaluation of changes in biochemical parameters with age of laying hens

The plasma concentrations of TG, TC, AST, ALT, FABP4, and LPL were measured in 40 laying hens every 5 weeks from age 27 to 52 weeks. The plasma concentrations of TG and TC displayed some variability among the individual layers. However, there was an increasing trend in TG levels with aging, compared to their concentrations in the laying hens at age 27 weeks (Figure 4a). At age 47 weeks, the concentrations of TG and TC were significantly higher than that at 32,37 , and 42 weeks. A significant decrease in the concentration of TC was observed in the plasma of the layers at age 52 weeks, compared to the obtained results at age 47 weeks, which was still higher than the obtained results of 27, 32, 37, and 42 weeks. No difference was found for the activities of ALT and AST among the six time points of 
the layers although the activities of ALT and AST tended to increase with age. According to the recommended weight range, the experimental chickens were overweight since age 32 weeks (Figure 4b). From 27 to 52 weeks of age, plasma TG and TC concentrations were significantly correlated with BW $(\mathrm{r}=0.55, \mathrm{P}<0.01$ and $\mathrm{r}=0.61, \mathrm{P}<$ 0.01 , respectively, Figure $4 \mathrm{c}$ ). This trend in BW was consistent with the increasing trends of $\mathrm{TG}$ and $\mathrm{TC}$ concentrations. Plasma TG concentrations were positively associated with TC $(\mathrm{r}=0.68, \mathrm{P}<0.01)$. The obtained results of ELISA revealed that FABP4 was significantly upregulated during 32-52 weeks of age (Figure 5a, P < $0.05)$. By age 52 weeks, the concentration of FABP4 and LPL was significantly higher than the age of 27 weeks (P $<0.05$ ).

\section{Correlation of FABP4 and LPL concentrations with} other variables

Plasma FABP4 was positively correlated with LPL, $\mathrm{TG}, \mathrm{TC}$, and $\mathrm{BW}(\mathrm{r}=0.29, \mathrm{r}=0.39, \mathrm{r}=0.20$, and $\mathrm{r}=0.31$, respectively, Figure $5 b)$. Other markers were significantly associated with each other (LPL vs BW, $r=0.18$; LPL vs $\mathrm{TC}, \quad \mathrm{r}=0.22 ;$ and LPL vs $\mathrm{TG}, \mathrm{r}=0.28)$. (a)

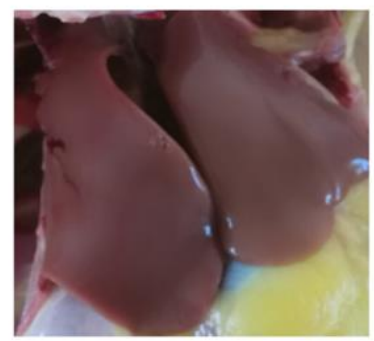

LW

(b)

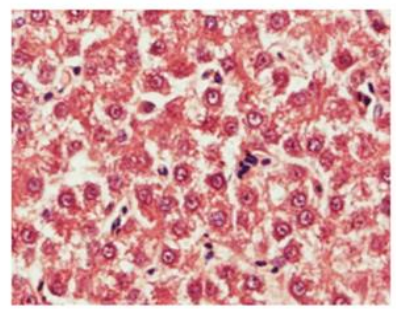

LW

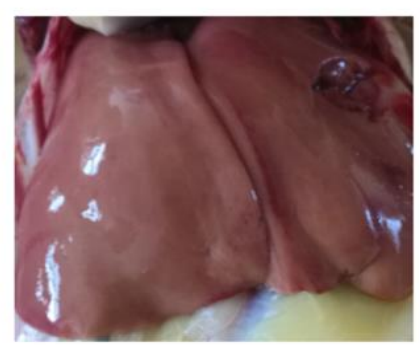

NW

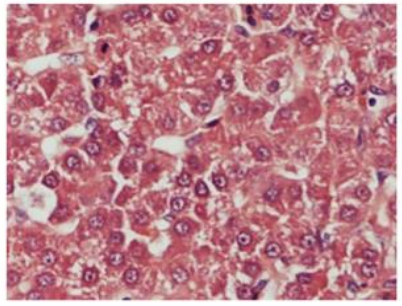

NW

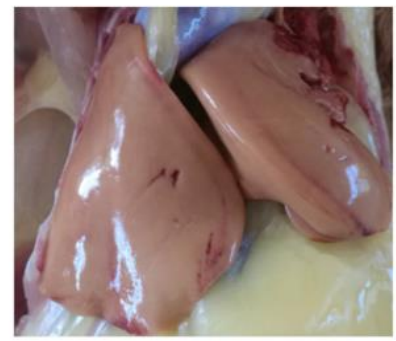

OW/Ob

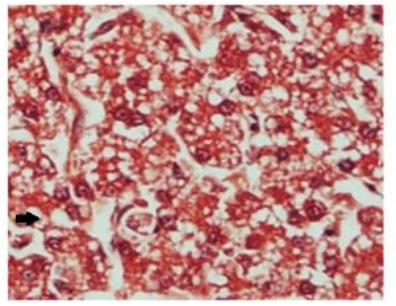

$\mathrm{OW} / \mathrm{Ob}$

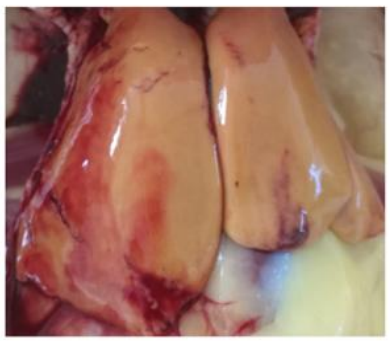

$\mathrm{SW} / \mathrm{Ob}$

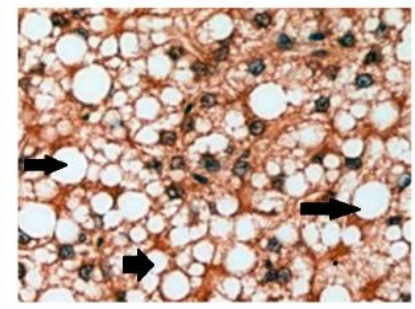

SW/Ob (c)

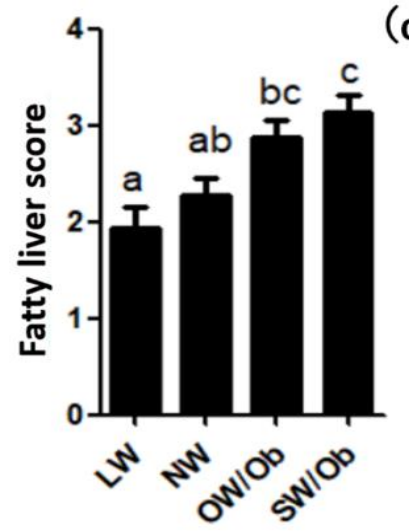

(d)

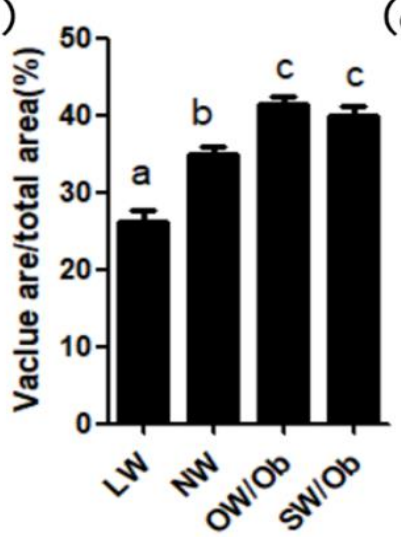

(e)

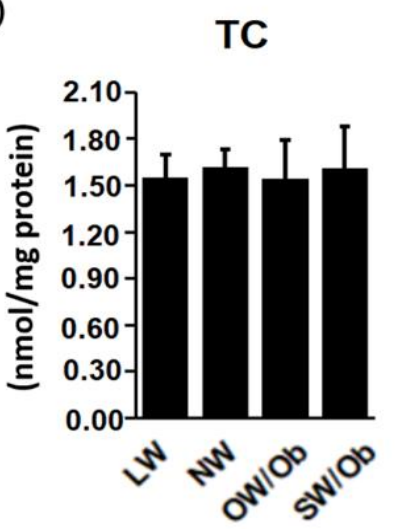

TG

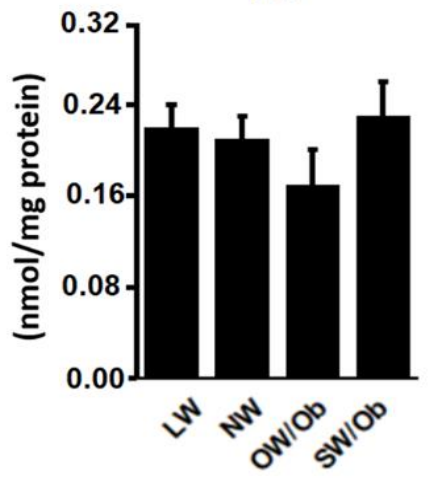

Figure 2. Results of evaluations of livers of chickens (52 weeks old) with different BWs. a: Representative images of various levels of liver hemorrhage. b: Representative images of histological sections of the liver after H\&E staining. The arrow indicates the steatosis and lipid vacuolization inside the hepatocytes. Photographs were taken at 400× magnification. c: Mean fatty liver score in chickens with different BWs (score 1: normal liver with dark red; 2: mild case of FLHS with mild yellow liver and hemorrhages; 3: moderate case of FLHS, light yellowish red liver and hemorrhages; 4: severe case of FLHS, large and massive hemorrhages with putty-colored livers). d: Quantified results of fat vacuoles area within the liver section. e: Liver TG and TC concentrations in chickens with different BW. LW: Low body weight, NW: Normal body weight, OW/Ob: Overweight/obese, SW/Ob: Seriously overweight/obese, ALT: Alanine aminotransferase, AST: Aspartate aminotransferase, TG: Triglyceride, TC: Total cholesterol. 
(a)

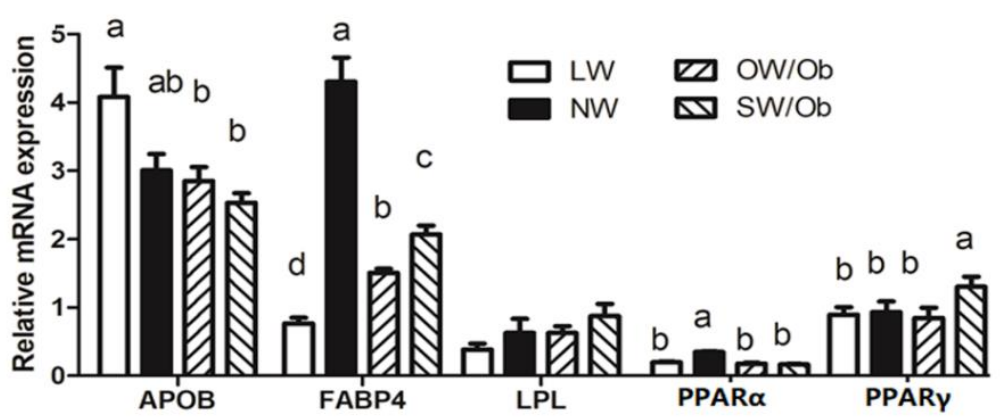

(b)

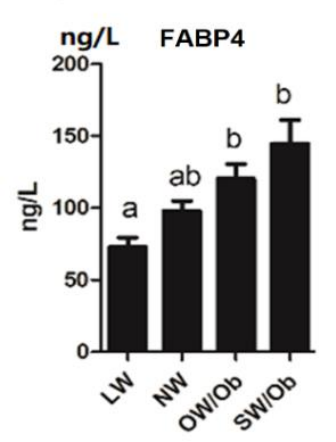

(c)
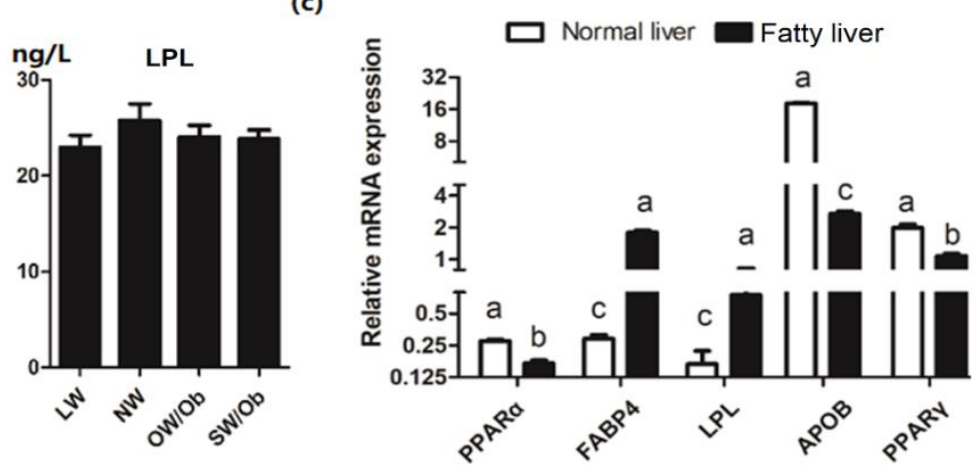

Figure 3. Evaluation of lipid metabolism-related parameters in relation to BW and liver condition. a: RT-qPCR analysis of lipid metabolism-related genes in livers of laying hens with different BW. b: Chicken plasma concentrations of FABP4 and LPL in laying hens with different BW ( $\mathrm{n}=15 / \mathrm{BW}$ group). c: RT-qPCR analysis of lipid metabolism-related genes in normal and fatty livers collected from laying hens of 27 and 52 weeks old, respectively. FABP4: Fatty acid-binding protein 4, LPL: Lipoprotein lipase, BW: Body weight, LW: Low body weight, NW: Normal body weight, OW/Ob: Overweight/obese, SW/Ob: Seriously overweight/obese.

(a)

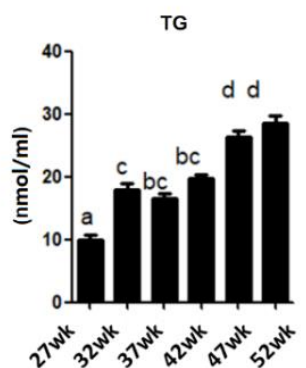

(b)

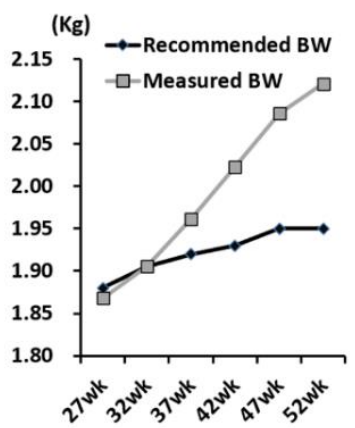

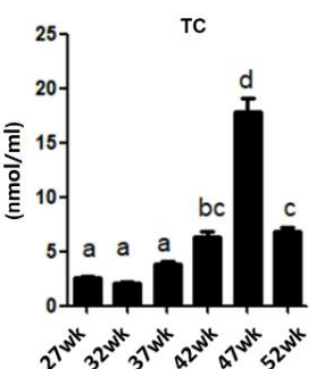

(c)

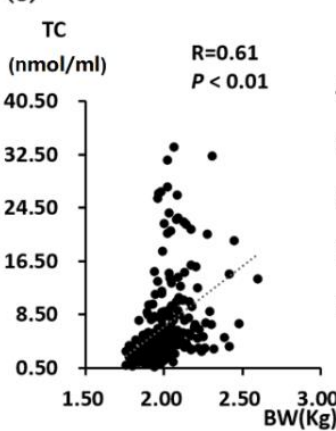

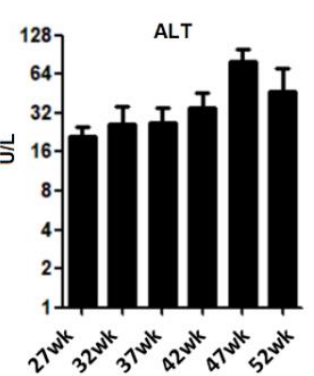

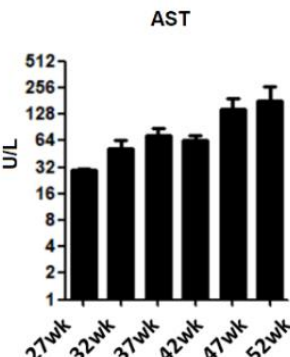

$(\mathrm{nmol} / \mathrm{ml})$

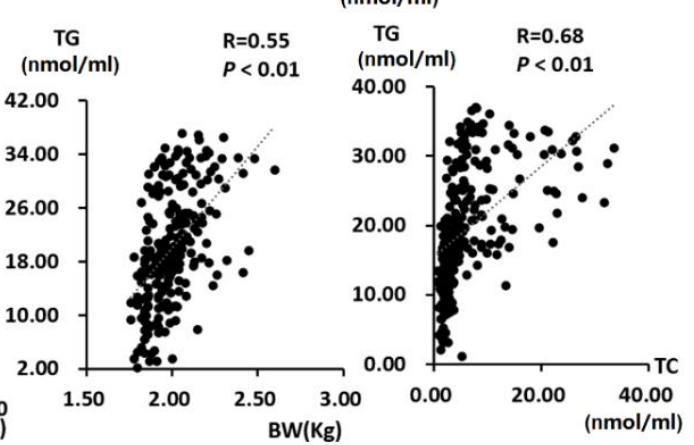

Figure 4. Evaluation of changes in biochemical parameters with age of laying hens. a: Plasma concentrations of TG, TC, ALT, and AST at six time points in laying hens. b: Comparison of measured BW of laying hens at different ages with recommended BW. c: Correlation of BW with TG and TC; Correlation of TG with TC. Values are expressed as mean \pm SEM. ALT: Alanine aminotransferase, AST: Aspartate aminotransferase, TG: Triglyceride, TC: Total cholesterol, FLHS: Fatty liver hemorrhagic syndrome, BW: Body weight. 
(b)

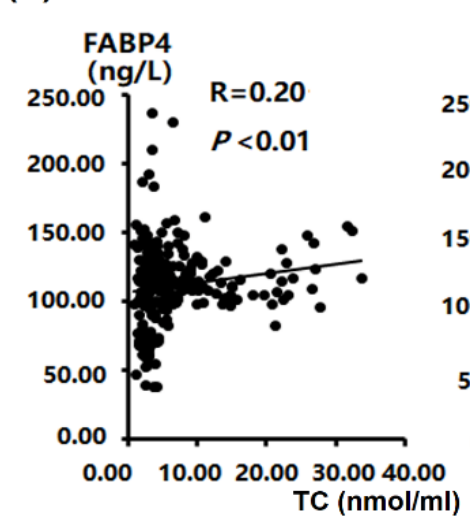

(a)
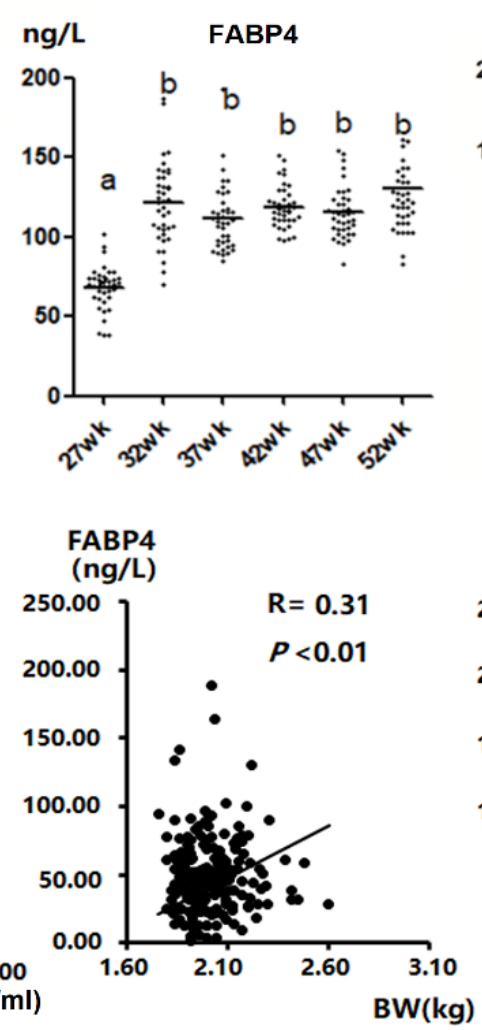
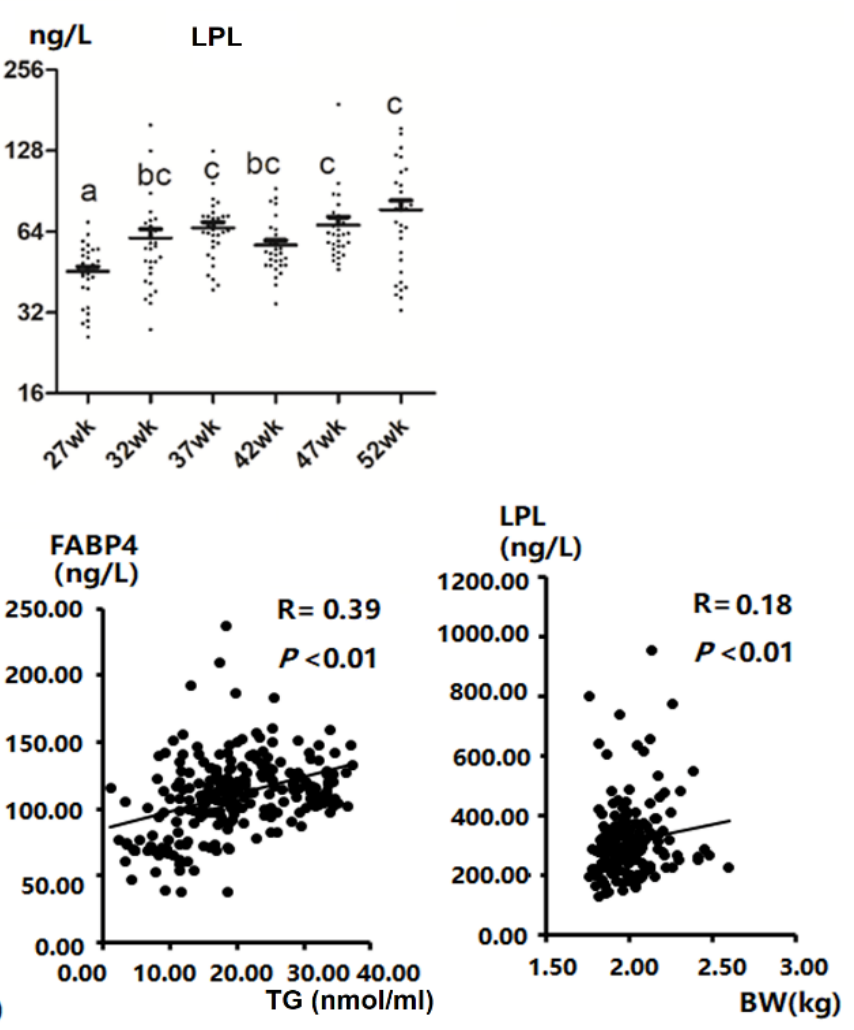
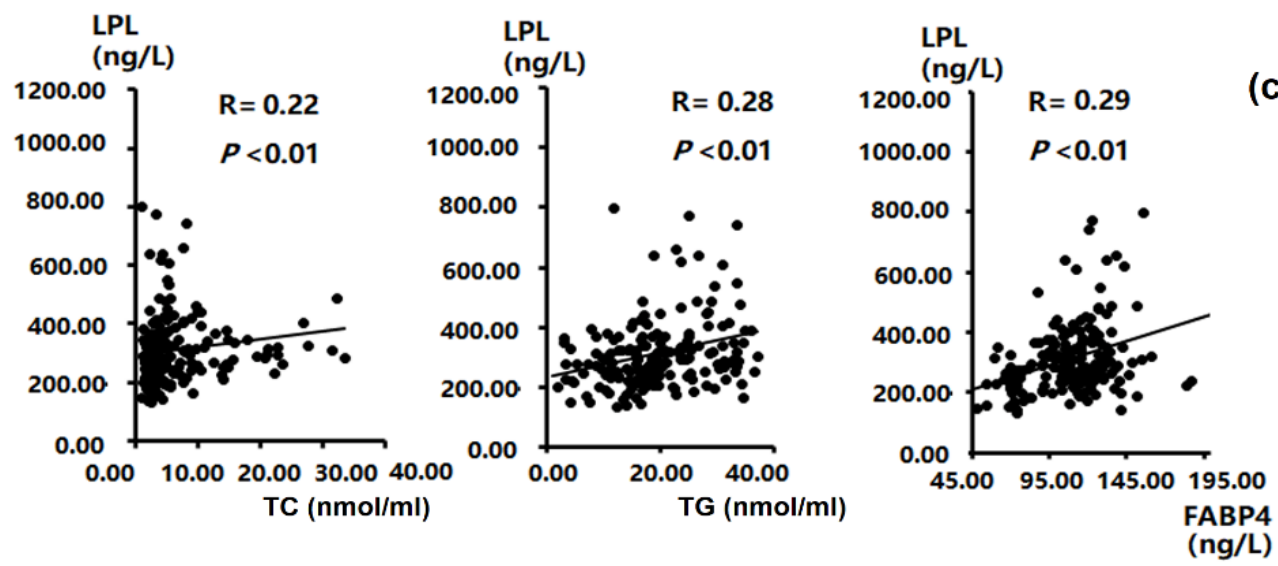

(c) FABP4

$(\mathbf{n g} / \mathrm{L})$

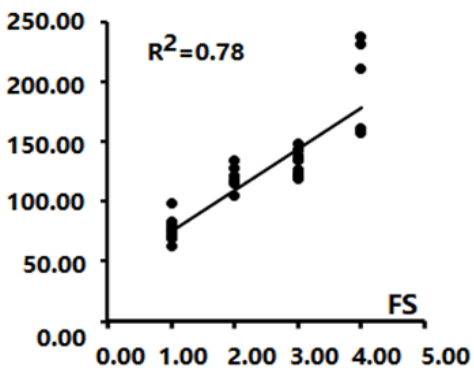

Figure 5. Evaluation of changes in FABP4 and LPL levels with age of laying hens. a: Scatter dot plot showing plasma concentrations of FABP4 and LPL in laying hens $(n=40)$ at different ages. b: Spearman's correlation analyses between FABP4 and LPL concentrations and other variables using data measured from 27 to 52 weeks of age. c: Correlation between plasma concentrations of FABP4 and FS of chickens (FS1: normal liver, $\mathrm{n}=13$; FS2: mild FLHS, $\mathrm{n}=12$; FS3: moderate FLHS, $\mathrm{n}=15$; FS4: severe FLHS, $\mathrm{n}=10$ ). FS: Fatty liver score, FABP4: Fatty acid-binding protein 4, LPL: Lipoprotein lipase, BW: Body weight.

\section{DISCUSSION}

FLHS is a metabolic disease characterized by excessive fat accumulation in the liver, accompanied by a hemorrhagic and pale liver, causing sudden death of laying hens (Shini et al., 2012). Signs of this metabolic disease are difficult to identify when the birds are alive, and it can only be determined through dissection. Therefore, it is urgent to find reliable noninvasive biomarkers to diagnose FLHS and monitor laying hens at an earlier time point when diet changes and potential health treatments can be used purposefully. The present study examined the relationship between BW and serum lipoproteins, and identified FABP4 as a potential novel circulating biomarker of FLHS. 
Based on findings addressing production, aged layers have an increased risk of FLHS, and the BW of these layers is always higher than recommended values in the breeding manual (Dong and Tong, 2019). Previous studies have indicated that the occurrence of FLHS is the result of abnormal accumulation of liver fat, mainly due to the large increase in liver TG, and the abnormal accumulation of fat caused by dysfunction in liver fat metabolism (Dong and Tong, 2019; Shini et al., 2019; Zhuang et al., 2019; Shini et al., 2020). The present study demonstrated that concentrations of TG, TC, and VLDL-C were positively correlated with BW in aged laying hens, and the amount of abdominal fat and the concentration of TG in aged layers significantly increased with BW gain. Lipid accumulation and hemorrhage were more severe in the overweight layers of the low BW group. These results suggested that the higher the weight, the higher the risk of FLHS in aged layers. However, it is worth noting that no difference was observed in the relative liver weight and the concentration of VLDL-C among chickens with different values of BW. The concentrations of TG were also similar among the $\mathrm{NW}$ and $\mathrm{OW} / \mathrm{Ob}$ group hens. Also, the relative weight of abdominal fat was similar between LW and NW hens. In addition, no significant difference in the fatty liver score was observed between layers in adjacent BW groups. Based on this, it can be found that the differences between layers in adjacent BW groups were relatively small, and BW within the standard range recommended by the manufacturer could not be used as an indicator of FLHS in aged layers. Thus, these findings indicated that BW of laying hens should be monitored at all times during production, but BW cannot be used as the sole criterion to discriminate aged laying hens with liver steatosis.

TG represent the main form of storage and transport of fatty acids in cells and circulating system, and they have been suggested to have the potential for diagnosis of NAFLD (Safaei et al., 2016). Evidence also suggests that TC is a risk factor for NAFLD, and as the level of TC improves, the incidence of NAFLD increases as well (Ballestri et al., 2016; Ren et al., 2019). High levels of TG and TC were also reported in previous studies in laying hens with FLHS (Dong and Tong, 2019; Gao et al., 2019). To find potential serum markers for early prediction of FLHS, changes in the plasma concentrations of TG and TC were examined every 5 weeks from 27 weeks (normal liver) to 52 weeks (FLHS was confirmed through dissection at the end of the experiment) of age in 40 laying hens. Furthermore, the serum activities of AST and ALT were examined during this period as liver injury indicators. It was found that TG and TC levels, which were positively associated with $\mathrm{BW}$, increased as chickens grew older. This means that TG and TC are good risk markers for FLHS although further confirmation is needed since the current study did not address the correlation of FLHS morphology with TG and TC levels at different periods. Furthermore, no difference was found in the activities of ALT and AST among the six time points, indicating that the classic liver injury indicators, AST and ALT, were unable to predict chicken FLHS, which was consistent with the conclusion drawn for NAFLD (Ekstedt et al., 2006). The results were also in line with a previous study conducted by Diaz et al. (1999) who found no clear correlation between plasma enzyme activities (AST, ALT, and lactate dehydrogenase) and the degree of liver hemorrhage in laying hens.

Finally, five known lipid metabolism-related genes, $A P O B, P P A R \alpha, P P A R \gamma, L P L$, and FABP4, were selected for mRNA expression analysis in fatty and normal livers. Expression of $A P O B, P P A R \alpha$, and PPAR $\gamma$ decreased in fatty livers, compared to the normal liver as opposed to the results reported by Li et al. (2015). They analyzed the liver transcriptome of young chickens (age 20 weeks) and laying hens (age 30 weeks) by sequencing, and found that the mRNA levels of fatty acid uptake and transport (PPAR,$A P O B$, and FABP3) increased in the liver of laying hens (Li et al., 2015). This difference may be due to the age of the birds studied. The current study examined the expression of lipid metabolism-related genes in the liver of aged chickens with FLHS, while Li et al. (2015) focused on younger birds at peak production. A similar result was reported by Song et al. (2017), in which obvious decreases in apolipoprotein (apo) A-I and apoB100 levels were observed in the hepatic tissues. The authors reported that expression of apoA-I decreased on day 60 while the apoB 100 levels slightly increased on day 30 and then decreased on day 60 in the liver of an FLHS model induced by a low-protein high-energy diet, and they speculated that this decrease was due to liver injury from FLHS (Song et al., 2017). APOB plays an important role in the hepatic export of triacylglycerols by interacting with TG, cholesterol, and phospholipids (Kessler et al., 2014; Devaraj and Jialal, 2020). The fatty acid oxidation related gene $P P A R \alpha$, together with $P P A R \gamma$ and $P P A R \beta / \delta$, is involved in many aspects of lipid metabolism (Bougarne et al., 2018). Hepatocyte PPAR $\alpha$ deletion impairs fatty acid catabolism resulting in hepatic lipid accumulation (Gao et al., 2015; Montagner et al., 2016). Hence, the downregulation of $A P O B$ and $P P A R \alpha$ may contribute to the hepatic lipid accumulation in the liver of aged laying hens with FLHS as suggested by Lu et al. (2019). 
The mRNA expression of FABP4 and $L P L$ significantly increased in the liver of FLHS chickens. FABP4 is an important lipid chaperone that plays an important role in lipid-mediated cellular physiological processes and metabolism. FABP4 is mainly expressed in adipocytes and macrophages, and high expression of FABP4 is closely related to the metabolic syndrome caused by obesity (Kralisch and Fasshauer, 2013). Elevated expression of hepatic FABP4 is positively correlated with the severity of NAFLD (Thompson et al., 2018). High levels of serum FABP4 are also reported in NAFLD patients, however, its use as a prognostic marker in serum is still controversial (Koh et al., 2009; Kim et al., 2011; Coilly et al., 2019). LPL transports plasma lipids into tissues and plays an important role in the regulation of lipid metabolism and energy balance (Li et al., 2014). In contrast, there is almost no LPL expression in normal adult liver, while increased hepatic $L P L$ mRNA expression is observed in obese humans, which is postulated to contribute to hepatic TG accumulation (Pardina et al., 2009). Consistent with the obtained results of NAFLD, mRNA analysis indicated that expression of FABP4 and $L P L$ was upregulated in the liver of laying hens with FLHS. Plasma FABP4 and LPL concentrations sequentially increased in chickens with increased $\mathrm{BW}$ indicating that plasma FABP4 and LPL might be risk markers for FLHS in laying hens. Accordingly, there was a positive correlation between the plasma level of the FABP4 and the severity of fatty liver in aged laying hens suggesting that FABP4 might be a potential diagnostic marker for FLHS.

\section{CONCLUSION}

In conclusion, plasma $\mathrm{TC}, \mathrm{TG}, \mathrm{FABP} 4$, and $\mathrm{LPL}$ are positively associated with BW, and risk of FLHS. Determination of these biomarkers in plasma samples from FLHS hens could provide a rapid and first-line diagnosis of FLHS for clinical application.

\section{DECLARATIONS}

\section{Acknowledgments}

This work was supported by the SAAS Program for Excellent Research Team (SPERT) under Grant (B-03) and China Agriculture Research System under Grant (CARS-40-K03).

\section{Competing interests} interests.

\section{Author contributions}

Professor CY supported the funding. Dr RL and CX did the RT-qPCR and ELISA assay. Mr NW, Mr GZ, and Ms YT collected the samples. Associate professor LZ designed the experiments, analyzed the data, did the experiments, and wrote the manuscript. All authors read and approved the final manuscript.

\section{REFERENCES}

Avinash Dhawale (2007). The liver: A big organ with a big role. World poultry, 23(10): 34-36. Available at: https://www.poultryworld.net/Breeders/Nutrition/2007/10/Theliver-A-big-organ-with-a-big-role-WP006905W/

Ballestri S, Nascimbeni F, Romagnoli D, and Lonardo A (2016). The independent predictors of non-alcoholic steatohepatitis and its individual histological features. Insulin resistance, serum uric acid, metabolic syndrome, alanine aminotransferase and serum total cholesterol are a clue to pathogenesis and candidate targets for treatment. Hepatology Research, 46(11): 1074-1087. DOI: http://www.doi.org/10.1111/hepr.12656

Bougarne NWB, Desmet SJ, Deckers J, Ray DW, Staels B, and De Bosscher K (2018). Molecular actions of $\operatorname{PPAR} \alpha$ in lipid metabolism and inflammation. Endocrine Reviews, 39(5): 760-802. DOI: http://www.doi.org/10.1210/er.2018-00064

Choi YI, Ahn HJ, Lee BK, Oh ST, An BK, and Kang CW (2012). Nutritional and hormonal induction of fatty liver syndrome and effects of dietary lipotropic factors in egg-type male chicks. AsianAustralasian Journal of Animal Sciences, 25(8): 1145-1152. DOI: http://www.doi.org/10.5713/ajas.2011.11418

Coilly A, Desterke C, Guettier C, Samuel D, and Chiappini F (2019). FABP4 and MMP9 levels identified as predictive factors for poor prognosis in patients with nonalcoholic fatty liver using data mining approaches and gene expression analysis. Scientific Reports, 9(1): 19785. DOI: http://www.doi.org/10.1038/s41598-019-56235-y

Devaraj S, and Jialal I (2020). Biochemistry, Apolipoprotein B. In StatPearls. StatPearls Publishing. Available at: https://www.ncbi.nlm.nih.gov/books/NBK538139/

Diaz GJ, Squires EJ, and Julian RJ (1999). The use of selected plasma enzyme activities for the diagnosis of fatty liver-hemorrhagic syndrome in laying hens. Avian Diseases, 43: 768-773. Available at: https://pubmed.ncbi.nlm.nih.gov/10611993/

Dong X, and Tong J (2019). Different susceptibility to fatty liverhaemorrhagic syndrome in young and older layers and the interaction on blood LDL-C levels between oestradiols and high energy-low protein diets. British Poultry Science, 60(3): 265-271. DOI: http://www.doi.org/10.1080/00071668.2019.1571164

Ekstedt M, Franzen LE, Mathiesen UL, Thorelius L, Holmqvist M, Bodemar G, and Kechagias S (2006). Long-term follow-up of patients with NAFLD and elevated liver enzymes. Hepatology, 44(4): 865-873. DOI: http://www.doi.org/10.1002/hep.21327

Gao Q JY, Yang G, Zhang X, Boddu PC, Petersen B, Narsingam S, Zhu YJ, Thimmapaya B, Kanwar YS, and Reddy JK (2015). PPAR $\alpha$ deficient ob/ob obese mice become more obese and manifest severe hepatic steatosis due to decreased fatty acid oxidation. American Journal of Pathology, 185(5): 1396-1408. DOI: http://www.doi.org/10.1016/j.ajpath.2015.01.018

Gao X LP, Wu C, Wang T, Liu G, Cao H, Zhang C, Hu G, and Guo X (2019). Effects of fatty liver hemorrhagic syndrome on the AMPactivated protein kinase signaling pathway in laying hens. Poultry $\begin{array}{lll}\text { Science, } & \text { 98(5): } 2201-2210 . & \end{array}$ http://www.doi.org/10.3382/ps/pey586

Hamid H ZJ, Li WX, Liu C, Li ML, Zhao LH, Ji C, and Ma QG (2019). Interactions between the cecal microbiota and non-alcoholic 
steatohepatitis using laying hens as the model. Poultry Science, 98(6): 2509-2521. DOI: http://www.doi.org/10.3382/ps/pey596

Julian RJ (2005). Production and growth related disorders and other metabolic diseases of poultry--a review. Veterinary Journal, 169(3): 350-369. DOI: http://www.doi.org/10.1016/j.tvjl.2004.04.015

Kessler EC, Gross JJ, Bruckmaier RM, and Albrecht C (2014). Cholesterol metabolism, transport, and hepatic regulation in dairy cows during transition and early lactation. Journal of Dairy Science, 97(9): 5481-5490. DOI: http://www.doi.org/10.3168/jds.2014-7926

Kim YC, Cho YK, Lee WY, Kim HJ, Park JH, Park DI, Sohn CI, Jeon WK, Kim BI et al. (2011). Serum adipocyte-specific fatty acidbinding protein is associated with nonalcoholic fatty liver disease in apparently healthy subjects. Journal of Nutritional Biochemistry, 22(3): 289-292. DOI: http://www.doi.org/10.1016/j.jnutbio.2010.02.007

Koh JH, Shin YG, Nam SM, Lee MY, Chung CH and Shin JY (2009). Serum adipocyte fatty acid-binding protein levels are associated with nonalcoholic fatty liver disease in type 2 diabetic patients. $\begin{array}{llll}\text { Diabetes } & \text { Care, } & 32(1) \text { : 147-152. } & \end{array}$ http://www.doi.org/10.2337/dc08-1379

Kralisch S, and Fasshauer M (2013). Adipocyte fatty acid binding protein: a novel adipokine involved in the pathogenesis of metabolic and vascular disease? Diabetologia, 56(1): 10-21. DOI: http://www.doi.org/10.1007/s00125-012-2737-4

Li H WT, Xu C, Wang D, Ren J, Li Y, Tian Y, Wang Y, Jiao Y, Kang X, and Liu $X$ (2015). Transcriptome profile of liver at different physiological stages reveals potential mode for lipid metabolism in laying hens. BMC Genomics, 16: 763. DOI: http://www.doi.org/10.1186/s12864-015-1943-0

Li Y, He PP, Zhang DW, Zheng XL, Cayabyab FS, Yin WD, and Tang CK (2014). Lipoprotein lipase: from gene to atherosclerosis. $\begin{array}{lll}\text { Atherosclerosis, } & \text { 237(2): }\end{array}$ http://www.doi.org/10.1016/j.atherosclerosis.2014.10.016

Livak KJ, and Schmittgen TD (2001). Analysis of relative gene expression data using real-time quantitative PCR and the 2 (-Delta Delta C(T)) Method. Methods, 25(4): 402-408. DOI: http://www.doi.org/10.1006/meth.2001.1262

Lu Z, He XF, Ma BB, Zhang L, Li JL, Jiang Y, Zhou GH, and Gao F (2019). Increased fat synthesis and limited apolipoprotein B cause lipid accumulation in the liver of broiler chickens exposed to chronic heat stress. Poultry Science,98(9): 3695-3704. DOI: http://www.doi.org/10.3382/ps/pez056

Montagner A, Polizzi A, Fouché E, Ducheix S, Lippi Y, Lasserre F, Barquissau V, Régnier M, Lukowicz C et al. (2016). Liver PPAR $\alpha$ is crucial for whole-body fatty acid homeostasis and is protective against NAFLD. Gut,65(7): 1202-1214. DOI: http://www.doi.org/10.1136/gutjnl-2015-310798

Pardina E, Baena-Fustegueras JA, Llamas R, Catalán R, Galard R, Lecube A, Fort JM, Llobera M, Allende H, Vargas V et al. (2009). Lipoprotein lipase expression in livers of morbidly obese patients could be responsible for liver steatosis. Obesity Surgery, 19(5): 608-616. DOI: http://www.doi.org/10.1007/s11695-009-9827-5

Ren XY, Shi D, Ding J, Cheng ZY, Li HY, Li JS, Pu HQ, Yang AM, He CL, Zhang JP et al. (2019). Total cholesterol to high-density lipoprotein cholesterol ratio is a significant predictor of nonalcoholic fatty liver: Jinchang cohort study. Lipids in Health and Disease, 18(1): 47. DOI: http://www.doi.org/10.1186/s12944-0190984-9
Rozenboim I, Mahato J, Cohen NA, and Tirosh O (2016). Low protein and high-energy diet: a possible natural cause of fatty liver hemorrhagic syndrome in caged White Leghorn laying hens. $\begin{array}{llll}\text { Poultry } & \text { Science, } & \text { 95(3): } & \text { 612-621. }\end{array}$ http://www.doi.org/10.3382/ps/pev367

Safaei A, Arefi Oskouie A, Mohebbi SR, Rezaei-Tavirani M, Mahboubi M, Peyvandi M, Okhovatian F, and Zamanian-Azodi M (2016). Metabolomic analysis of human cirrhosis, hepatocellular carcinoma, non-alcoholic fatty liver disease and non-alcoholic steatohepatitis diseases. Gastroenterology and Hepatology from Bed to Bench, 9(3), 158-173. Available at: https://pubmed.ncbi.nlm.nih.gov/27458508/

Shini A, Shini S, Filippich LJ, Anderson ST, and Bryden WL (2012). Role of inflammation in the pathogenesis of fatty liver haemorrhagic syndrome in laying hens. Proceedings of the Australian Poultry Science Symposium. University of Sydney Poultry Research Foundation. Available at: https://espace.library.uq.edu.au/view/UQ:272199

Shini S, Shini A, and Bryden WL (2019). Unravelling fatty liver haemorrhagic syndrome: 2. Inflammation and pathophysiology. Avian Pathology, 49(2): 131-143. DOI: http://www.doi.org/10.1080/03079457.2019.1682119

Shini S, Shini A, and Bryden WL (2020). Unravelling fatty liver haemorrhagic syndrome: 1. Oestrogen and inflammation. Avian Pathology,49(1): 87-98. DOI: http://www.doi.org/10.1080/03079457.2019.1674444

Song Y, Ruan J, Luo J, Wang T, Yang F, Cao H, Huang J, and Hu G (2017). Abnormal histopathology, fat percent and hepatic apolipoprotein A I and apolipoprotein B100 mRNA expression in fatty liver hemorrhagic syndrome and their improvement by soybean lecithin. Poultry Science, 96(10): 3559-3563. DOI: http://www.doi.org/10.3382/ps/pex163

Thompson KJ, Austin RG, Nazari SS, Gersin KS, Iannitti DA, and McKillop IH (2018). Altered fatty acid-binding protein 4 (FABP4) expression and function in human and animal models of hepatocellular carcinoma. Liver International : Official Journal of the International Association for the Study of the Liver, 38(6): 1074-1083. DOI: http://www.doi.org/10.1111/liv.13639

Trott KA, Giannitti F, Rimoldi G, Hill A, Woods L, Barr B, Anderson M, and Mete A (2014). Fatty liver hemorrhagic syndrome in the backyard chicken: a retrospective histopathologic case series. Veterinary Pathology, 51(4): 787-795. DOI: http://www.doi.org/10.1177/0300985813503569

Vilar-Gomez E, and Chalasani N (2018). Non-invasive assessment of non-alcoholic fatty liver disease: Clinical prediction rules and blood-based biomarkers. Journal of Hepatology, 68(2): 305-315. DOI: http://www.doi.org/10.1016/j.jhep.2017.11.013

Whitehead CC (1979). Nutritional and metabolic aspects of fatty liver disease in poultry. The Veterinary Quarterly, 1(3): 150-157. DOI: http://www.doi.org/10.1080/01652176.1979.9693738

Wolford JH, and Polin D (1974). Induced fatty liver-hemorrhagic syndrome (FLHS) and accumulation of hepatic lipid in force-fed laying chickens. Poultry Science, 53(1): 65-74. DOI: http://www.doi.org/10.3382/ps.0530065

Zhuang Y, Xing C, Cao H, Zhang C, Luo J, Guo X, and Hu G (2019). Insulin resistance and metabonomics analysis of fatty liver haemorrhagic syndrome in laying hens induced by a high-energy low-protein diet. Scientific Reports, 9(1): 10141. DOI: http://www.doi.org/10.1038/s41598-019-46183-y 